\title{
Kinetics of deposition and clearance of inhaled mineral dusts during chronic exposure
}

\author{
J H VINCENT, A M JOHNSTON, A D JONES, R E BOLTON, AND J ADDISON \\ From the Institute of Occupational Medicine, Edinburgh EH89SU, UK
}

ABSTRACT New inhalation studies have been carried out with rats exposed to UICC (Union International Contre le Cancer) amosite asbestos, with the main aim of further elucidating the factors that influence the accumulation of dust in the lung during prolonged chronic exposure. The results show that, for exposure times beyond a few weeks, the lung burden rises linearly and does not level off as predicted by simple models based on ideas taken from the 1966 report of the Task Group on Lung Dynamics. Furthermore, the lung burden is found to scale directly in proportion to the exposure concentration in a way that seems to contradict the overload hypothesis stated earlier. Nevertheless, the general pattern exhibited by our results for asbestos is markedly similar to that found elsewhere for rats inhaling diesel fume, leading to $(a)$ the suggestion that it is general (and not specific to fibrous dust); and (b) the hypothesis that, whereas overload of clearance can take place at high lung burdens after exposure has ceased, it is cancelled by the sustained stimulus to clearance mechanisms provided by the continuous challenge of chronic exposure. The linearity of the increase in lung burden is explained in terms of a kinetic model involving sequestration of some inhaled material to parts of the lung where it is difficult to clear. The particular sequestration model favoured is one where, the longer a particle remains in the lung without being cleared, the more likely it will be sequestrated (and therefore less likely cleared). It is believed that such ideas may eventually be useful in forming exposure-dose relations for epidemiology.

In associating respiratory disease with exposure to airborne dust we are concerned, firstly, with the inhalation and deposition of particles in the lung during the act of breathing and, secondly, with the clearance of those particles from the lung. Taken together, these processes govern the cumulative dust dose experienced by the lung. The kinetics of deposition and clearance are therefore of primary importance in the relation between exposure and cumulative dose, and are therefore ultimately fundamental to the biological processes leading to disease.

While the present paper is drawn from experimental studies with rats involving asbestos dusts (since this is where much of our work so far has been directed), many of the conclusions reached are believed to be pertinent to a wide range of other relatively insoluble dusty materials. Particular attention is given to the problem of the accumulation of lung burden during prolonged chronic exposure, relevant to the exposure of workers to mineral dusts in a wide range of industries.

Received 19 November 1984 Accepted 18 February 1985

\section{Background}

Mathematical models of lung clearance do not set out initially to describe mechanistically the complex biological processes that take place in the respiratory system. Multicompartmental models of the type described by the Task Group on Lung Dynamics ${ }^{1}$ relate only loosely to the actual anatomical structure of the lung. Rather they are, in the first instance, consequences of the fact that any monotonically falling curve (such as that describing the temporal changes in the retention of material in the lung after a period of inhalation) may be represented by a series of superposed exponential functions. Therefore the concept of dividing the lung into compartments is notional, aimed-particularly with regard to radioactive dusts - at providing a basis for dosimetry estimates. Such models can, however, serve a useful secondary purpose in helping to test biological hypotheses for lung responses to inhaled particles.

The simplest mathematical representation is based on the working assumption that the rate at which inhaled material is being cleared instantaneously from a given compartment is directly proportional to the 


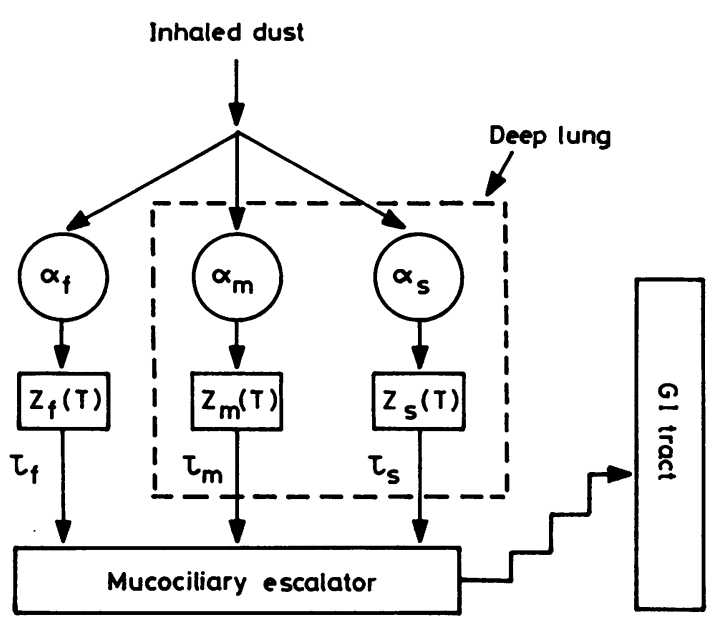

Fig 1 Schematic of simple lung kinetic model.

amount which is present. For chronic exposure to airborne dust, the kinetics of the accumulation of material in the compartment are therefore described by:

$$
\mathrm{dZ}(\mathrm{t})=\alpha \mathrm{dt}-\frac{\mathrm{Z}(\mathrm{t})}{\tau} \mathrm{dt}
$$

where $Z(t)$ is the lung burden per unit respirable dust exposure concentration at time $t$ since the beginning of exposure, $\alpha$ the amount deposited per unit dust concentration per unit time, and $1 / \tau$ a constant characteristic of the clearance rate. Assuming that $\alpha$ is constant regardless of the age of the subject and that $\tau$ is constant independent of the level of exposure or the lung burden which is present, integration of equation (1) yields the cumulative lung burden at time $\mathrm{t}=\mathrm{T}$ :

$$
Z(T)=\alpha \tau[1-\exp (-T / \tau)]
$$

where it is now seen that $\tau$ is the time constant of the exponential clearance process for the compartment and is related to the more familiar clearance half time $\tau_{1 / 2}$ by

$$
\tau_{1 / 2}=0.693 \tau \text {. }
$$

If exposure ceases at time $T$ then the lung burden at time $\Delta \mathrm{T}$ postexposure is given by:

$$
\mathrm{Z}(\mathrm{T}, \Delta \mathrm{T})=\alpha \tau[1-\exp (-\mathrm{T} / \tau)] \exp (-\Delta \mathrm{T}) .
$$

To obtain the cumulative burden for the whole lung, we add together all the $\mathrm{Zs}$ for the various individual compartments.

The constants in the above equations may be estimated by short term inhalation experiments. For example, the results of Morgan et al for an aerosol of a synthetic fluoramphibole, inhaled by rats in air- borne respirable concentrations of up to $50 \mathrm{mg} / \mathrm{m}^{3}$ for $\overline{\bar{\alpha}}$ just 30 minutes followed by the monitoring of reten- $\bar{z}$ tion for up to 120 days, ${ }^{2}$ are consistent with a simple $\stackrel{\odot}{\stackrel{\odot}{~}}$ model having three compartments (nominally "fast," "medium," and "slow" clearing) represented by clearance time constants $(\tau)$ of about $0 \cdot 6,11 \cdot 5$, and $170 \tau$ days respectively (with corresponding $\tau_{1 / 2}$ values of $\frac{C}{\partial}$ $0.4,8$, and 118 days). This model is shown sche- $\overline{\bar{c}}$. matically in fig 1 , where it is assumed that the partic- $\vec{\phi}$ ulate material in question is insoluble in body fluids. Middleton et al reported results for UICC (Union $\infty$ International Contre le Cancer) amosite which also $\overrightarrow{0}$ are in broad agreement with this picture. They went $\vec{\overrightarrow{ }}$ on to suggest that, biologically, the first of these three $\omega_{\sigma}$ compartments may be broadly related to the clearance $O$ of particles deposited directly in the ciliated airways $\frac{\mathbb{D}}{3}$ and so removed relatively rapidly through the mucociliary escalator and, subsequently, the gastro- ?

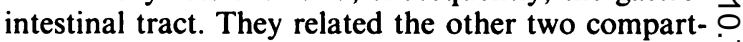
ments to the clearance of particles deposited in the $\partial$ non-ciliated respiratory bronchioles and alveoli by the phagocytic actions of pulmonary macrophages, with $\mathrm{O}$ the difference in clearance time constant between the $\vec{O}$ two compartments being associated with the degree of "fixation" of the deposited dust.

More recently we have reported inhalation studies $\mathbb{\complement}$ with UICC amosite ${ }^{5}$ in which the build up of material $\vec{\bullet}$ in the deep lungs of rats (nominally, the two slower clearing compartments) was obtained as a function of

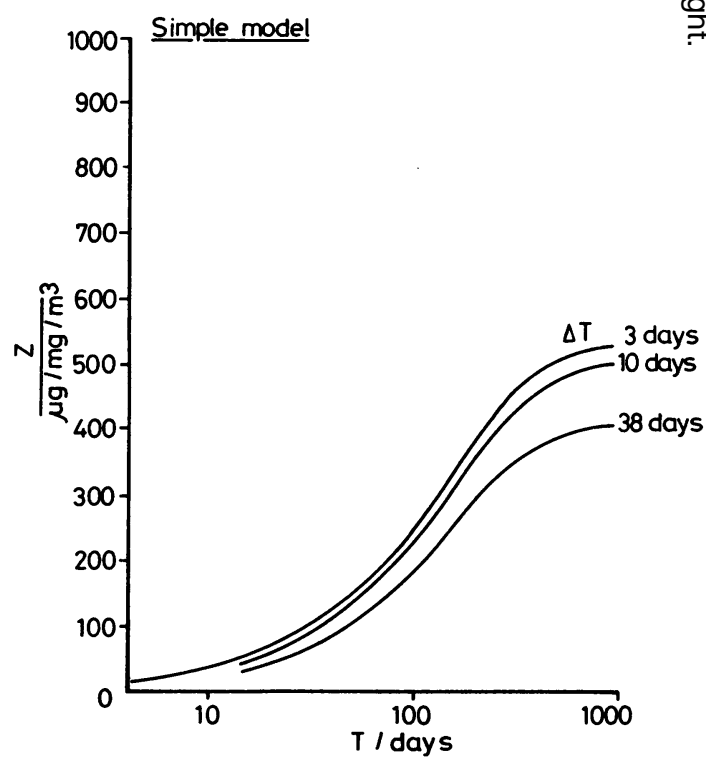

Fig 2 Predictions of lung burden during chronic exposure. as a function of exposure time (T) for various postexposure times $(\Delta T)$, using parameters derived from short term inhalation studies with rats using UICC amosite. ${ }^{235}$ 
time during chronic exposure of relatively short duration up to 40 days. Using $\tau_{m}=11.5$ and $\tau_{s}=170$ days (where the subscripts $m$ and $s$ to these time constants relate to the medium and slow clearance compartments respectively), good agreement between theory and experiment was achieved by the choices of $\alpha_{\mathrm{m}}=2.80$ and $\alpha_{\mathrm{s}}=3.05 \mu \mathrm{g} / \mathrm{mg} / \mathrm{m}^{3} /$ day (averaged over the weekly dusting cycle). From the data given by Morgan et al for the lung regional deposition distribution ratios for the synthetic fluoramphibole (assumed to be similar to those for the UICC amosite), ${ }^{2}$ together with estimates of the breathing volumes of the animals under the conditions of exposure, these are seen to be plausible. The simple kinetic model may be used, incorporating these values for the $\alpha s$ and $\tau s$, to calculate the accumulation of deep lung burden of UICC amosite during more prolonged chronic exposure. The results are shown in fig 2 , the most important feature of which is the prediction that the accumulation of lung burden eventually tends to level off, reaching about $95 \%$ of its final value after about one year.

Previously ${ }^{5}$ it was suggested, from the results of our 40 day chronic inhalation study and those of Wagner et $a l^{6}$ and Davis $e t a l^{7}$ for periods of exposure of up to two years, that such levelling off does not occur in practice. Rather, a steadily increasing lung burden was indicated. By pronounced contrast, the results of Wagner et al for UICC chrysotile, where corresponding cumulative lung burdens were much lower, exhibited a trend more consistent with the model. ${ }^{6}$ This combined evidence led us to postulate that, at high enough lung burden, overload of clearance takes place, leading to enhancement of the rate of increase of lung burden. Studies of clearance after short term inhalations at various exposure concentrations ${ }^{5}$ supported this by indicating a significantly reduced rate of clearance for lung burdens exceeding about $1 \mathrm{mg}$.

Such an idea of clearance overload is not new, similar hypotheses having been previously suggested by several other workers to explain apparently impaired clearance of aerosols inhaled at concentrations similar to those in the work already cited (see, for example, Ferin $^{8}$ in relation to the quartz studies of Klosterkotter and Bunemann ${ }^{9}$ and Vostal et al $^{10}$ in relation to their studies of diesel fume inhalation). This paper describes new chronic exposure experiments which have been carried out using UICC amosite to enable closer examination of this and other associated ideas.

\section{Experimental}

All the experiments were carried out using outbred male SPF Wistar rats of the AF/HAN strain, exposed to airborne dust of UICC amosite in $1 \mathrm{~m}^{3}$ inhalation chambers in separate groups of 12 animals (with up to
48 per chamber at any given time). The rats were all older than eight months (for which it had been established that systematic variations in lung burden with age are weak). Details of the dusting facilities and procedures have been fully described elsewhere. ${ }^{3411}$ The dusting regimen and the times of insertion into the chambers of the individual groups of rats were chosen so that the animals could be exposed for durations of up to one year (for seven hours a day on five days a week). Dust exposure levels were defined for each experiment in terms of the running day to day averages of respirable dust concentration as measured using Casella type 113A gravimetric samplers. Two parallel experiments were conducted, one at the target respirable dust concentration of $1 \mathrm{mg} / \mathrm{m}^{3}$ and the other at $10 \mathrm{mg} / \mathrm{m}^{3}$.

Animals were removed in the groups of 12 after chosen exposure times $(T)$ and killed in subgroups of four at postexposure times $(\Delta T)$ of three, 10 , and 38 days respectively. After the lungs (including the associated mediastinal and hilar lymph nodes) were excised in toto, the burdens of accumulated dust were recovered and assessed by the plasma ashing/infrared spectrophotometric method described elsewhere. ${ }^{35}$ The choice of the above $\Delta \mathrm{T}$-values ensured that, to all

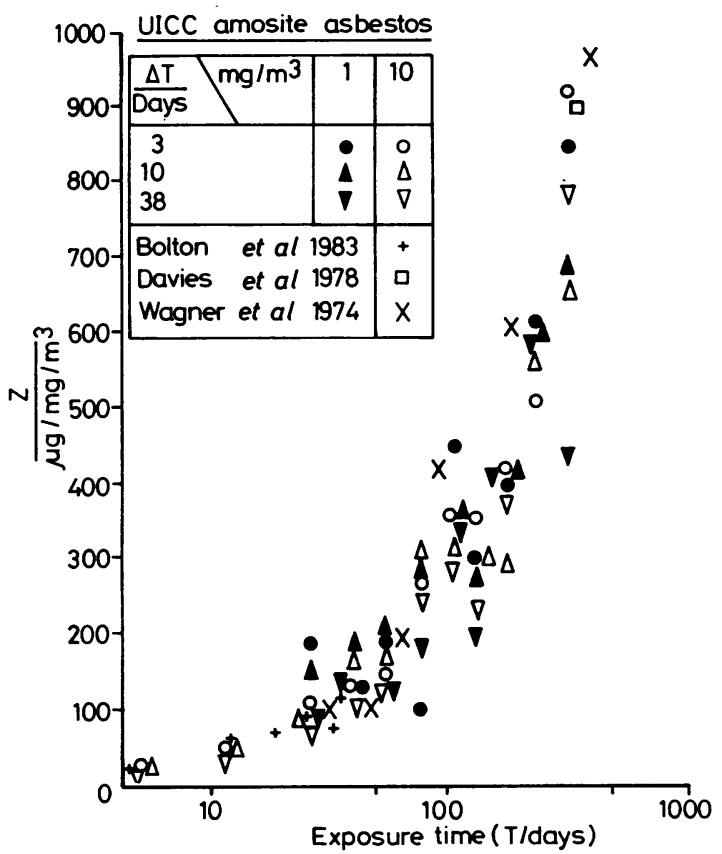

Fig 3 Measurements of lung burden in rats during chronic exposure as a function of exposure time $(T)$ for various postexposure times $(\Delta T)$ and for various exposure concentrations. Data are also shown from previously reported studies. ${ }^{5-7}$ 
intents and purposes, the measured lung burdens related to material deposited in the deep lung compartments only.

\section{Results}

The results are presented in fig 3 in the form suggested by equation (4), with $Z(T, \Delta T)$ representing the measured overall deep lung burden normalised with respect to respirable dust concentration. In attempts to reduce the scatter in the data points the possibility was examined of further normalising the results with respect to the body weights, wet lung weights, or dry lung weights, or a combination of these, of the individual animals. None of these brought about any perceptible reduction in the variability, however, and so this idea was discarded. Also included in fig 3 are the earlier data of Wagner et al, ${ }^{6}$ Davis et al, ${ }^{7}$ and Bolton et al. ${ }^{5}$ Within the variability indicated, the small systematic differences between the individual data sets for each of the postexposure times $(\Delta T)$ broadly reflect the clearance pattern predicted by equation (4).

Figure 3 shows several interesting features. Firstly, the results from the various experiments-some of them from a different laboratory using different analytical methods-display encouraging general agreement. Secondly, the results for the 1 and $10 \mathrm{mg} / \mathrm{m}^{3}$ exposure levels, when plotted in the form shown, appear to collapse into a unique relationship. This suggests that the kinetics of the clearance process are independent of the magnitude of lung burden. In this respect, therefore, the results are qualitatively consistent with the assumptions underlying the simple model. Thirdly, however, while the results are in good quantitative agreement with the simple theory (see fig

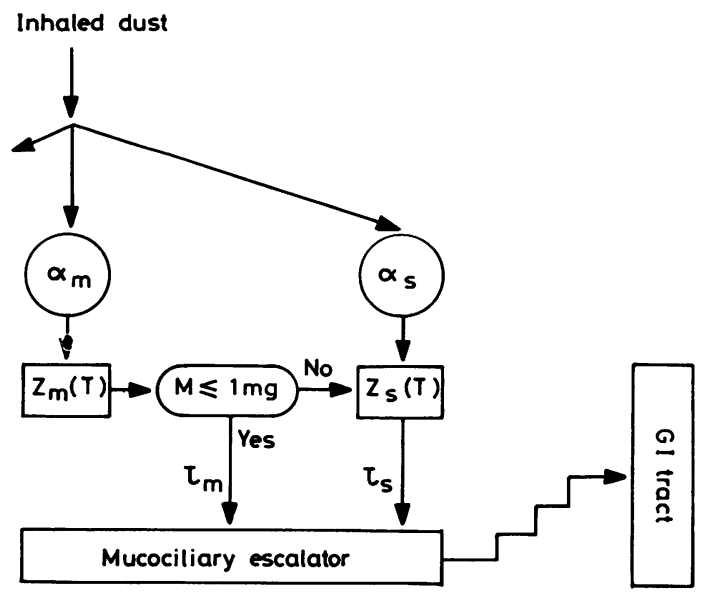

Fig 4 Schematic of lung kinetic model for clearance overload.
1) during the early weeks of exposure, they are seen later to be in progressively greater disagreement, with the lung burdens being much larger than predicted. In particular, there is no evidence of the predicted levelling off, confirming the experimental trends reported in our earlier paper. ${ }^{5}$ In fact, inspection of the results shows that, for $\mathrm{T}>40$ days, the relation between $\mathrm{Z}(\mathrm{T})$ and $\mathrm{T}$ (lumping together the results for all the $\Delta T s)$ is strongly linear, with

$$
\begin{aligned}
\mathrm{Z}(\mathrm{T}) & =2.05 \mathrm{~T}+60, \\
& \text { correlation coefficient }=0.92 .
\end{aligned}
$$

Clearly the simple kinetic model based on the Task Group on Lung Dynamics ${ }^{1}$ does not account for such a trend, and therefore an alternative model is required.

\section{Alternative kinetic models}

\section{OVERLOAD}

In our overload hypothesis it was suggested that clearance proceeds during the early stages of exposure according to the simple theory until the magnitude of the deep lung burden builds up to a level where a breakdown of some of the clearance mechanisms takes place. ${ }^{5}$ In its simplest form, supported by our clearance studies reported in that paper, the transition may take the form of a sudden and complete failure of the medium clearance compartment and therefore transfer of all material in the deep lung into the slowe clearance compartment. This model may be repre-? sented schematically by fig 4 and mathematically by

$$
\begin{aligned}
\mathrm{Z}(\mathrm{T}, \Delta \mathrm{T}) & =\alpha_{\mathrm{m}} \tau_{\mathrm{m}}\left[1-\exp \left(-\mathrm{T} / \tau_{\mathrm{m}}\right)\right] \exp \left(-\Delta \mathrm{T} / \tau_{\mathrm{m}}\right) \\
& +\alpha_{\mathrm{s}} \tau_{\mathrm{s}}\left[1-\exp \left(-\mathrm{T} / \tau_{\mathrm{s}}\right)\right] \exp \left(-\Delta \mathrm{T} / \tau_{\mathrm{s}}\right)
\end{aligned}
$$

for $T<T^{\prime}$ (the time at which the deep lung burden reaches the threshold for overload), and by

$$
\begin{aligned}
& \mathrm{Z}(\mathrm{T}, \Delta \mathrm{T})=\mathrm{Z}\left(\mathrm{T}^{\prime}\right) \exp \left[-\left(\mathrm{T}-\mathrm{T}^{\prime}\right) / \tau_{\mathrm{s}}\right] \exp \left(-\Delta \mathrm{T} / \tau_{\mathrm{s}}\right) \\
& +\left(\alpha_{\mathrm{m}}+\alpha_{\mathrm{s}}\right) \tau_{\mathrm{s}}\left\{1-\exp \left[-\left(\mathrm{T}-\mathrm{T}^{\prime}\right) / \tau_{\mathrm{s}}\right]\right\} \exp \left(-\Delta \mathrm{T} / \tau_{\mathrm{s}}\right)
\end{aligned}
$$

for $\mathrm{T}>\mathrm{T}^{\prime}$.

Figure 5 shows the behaviour of $\mathrm{Z}(\mathrm{T})$ calculated from this model, taking $\Delta T=10$ days and assuming that the threshold lung burden for overload of clearance is $1 \mathrm{mg}$. This latter choice is based on the results of our own short term clearance studies reported earlier ${ }^{5}$ for UICC amosite, of Klosterkotter and Bunemann for quartz, ${ }^{9}$ and of Vostal et al for diesel fume. ${ }^{10}$ In fig 5 the results of the overload model calculations (solid lines) are compared directly with the experimental data. For $10 \mathrm{mg} / \mathrm{m}^{3}$, agreement is certainly better than for the simple kinetic model (dashed line). For $1 \mathrm{mg} / \mathrm{m}^{3}$, however, the predicted curve merely follows the simple model since the the- 


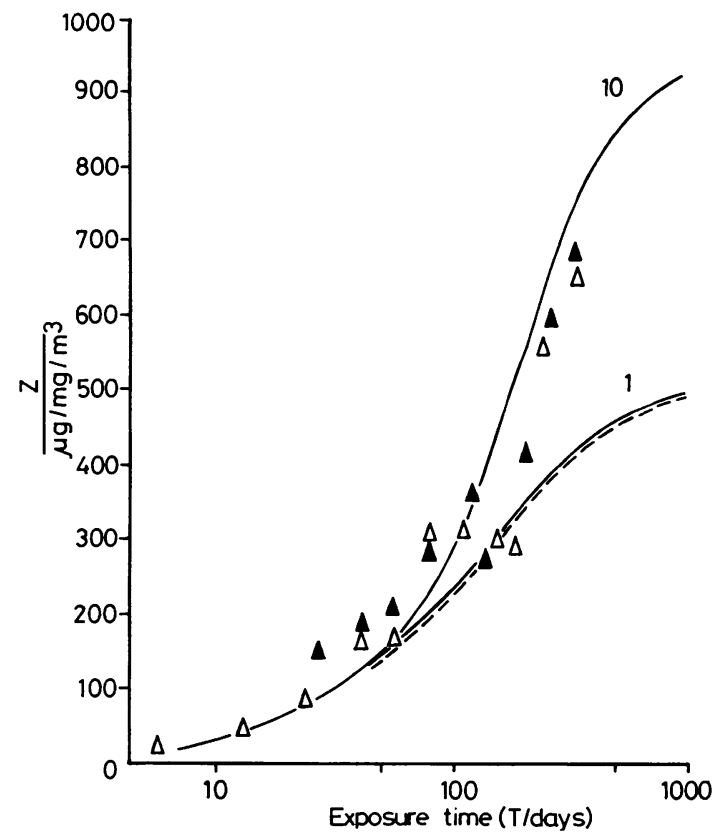

Fig 5 Results of predictions of lung burden during chronic exposure using "overload" kinetic model with parameters derived from short term studies with rats inhaling UICC amosite $^{235}$ (solid line). Calculations are compared with experimental data for exposure concentrations of 1 and 10 $\mathrm{mg} / \mathrm{m}^{3}$ respectively, and for postexposure time $(\Delta T) 10$ days. Predictions based on simple model are shown, for comparison purposes, in form of dashed line.

oretical lung burden never reaches the $1 \mathrm{mg}$ threshold. The predicted divergence between the trends for the two contrasting dust exposure levels is not reflected in the experimental data. There are, of course, other ways in which such an overload hypothesis may be represented mathematically. Nevertheless, the end result will be broadly similar. Therefore, despite the substantial evidence (from results of clearance after short term inhalation exposures from a number of sources) for the slowing of deep lung clearance at high lung burdens, it appears that such an overload phenomenon is not predominantly responsible for the main trends in cumulative lung burden observed during prolonged chronic exposure. We will return to this apparent contradiction later in the paper.

\section{SEQUESTRATION}

Other workers also have recognised the inadequacy of the simple kinetic model for describing the accumulation of inhaled material in the lung during prolonged chronic exposure. In experience markedly similar to our own, workers at General Motors
Laboratories (Vostal et al, ${ }^{10}$ Chan et al ${ }^{12}$ ) discovered that the prediction of cumulative lung burden of diesel fume particulate in rats, using the simple kinetic model with parameters derived from short term clearance studies, agreed well with the experimental chronic exposure results at short exposure times but failed to account for the continuing steady rise at longer exposure times.

It has long been recognised that clearance of particles from the alveolar interstitium and from within certain areas of pathological change may be particularly difficult..$^{91314}$ Soderholm proposed (at the EPA Diesel Emissions Symposium, Raleigh, North Carolina, 1981) a modified multicompartmental kinetic model, the key feature of which is a sequestration compartment (in addition to lymph nodes) into which material flows from other deep lung compartments but from which itself there is no clearance (or, at least, from which clearance is slow). The consequence of such a model is that, at long exposure durations for which the accumulations of material in the finitely clearing compartments have eventually levelled off, there ultimately remains a linear accumulation of material in the sequestration compartment. Initial support for this view is provided by the results of recent experimental studies-for instance, Morgan et al for fibrous dusts in rats, ${ }^{15}$ Snipes et al for nonfibrous dusts in rodents and dogs, ${ }^{16}$ and Bailey et al for non-fibrous dusts in rodents and $\operatorname{man}^{1718}$ in which clearance after short term inhalations was examined for periods postexposure substantially longer than the 120 days of the studies cited earlier. These all show that clearance tends to become progressively slower, even (in some cases) to the point where lung retention

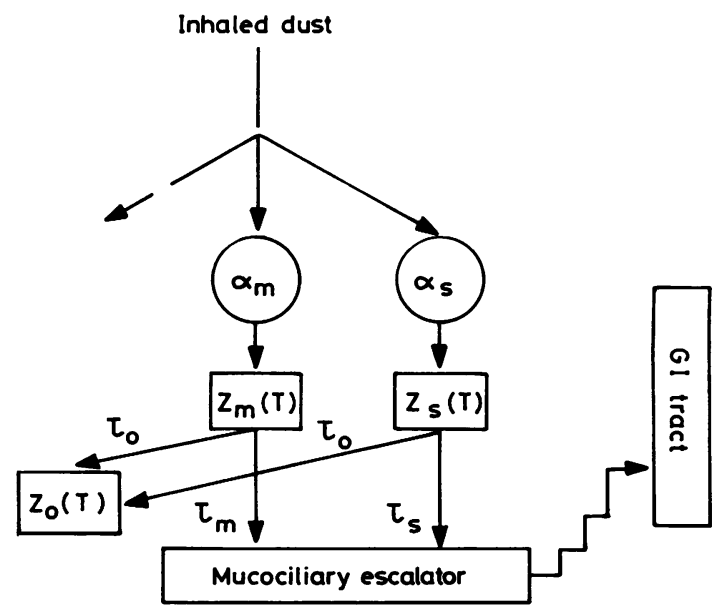

Fig 6 Schematic of lung kinetic model for sequestration. 
becomes asymptotic to finite values greater than zero (as high as $20 \%$ ).

Following these basic ideas, we can construct a set of kinetic equations representing the model shown schematically in fig 6 , thus

$$
\begin{aligned}
& d Z_{m}(t)=\alpha_{m} d t-\frac{Z_{m}(t)}{\tau_{m}} d t-\frac{Z_{m}(t)}{\tau_{o m}} d t \\
& d Z_{s}(t)=\alpha_{s} d t-\frac{Z_{s}(t)}{\tau_{s}} d t-\frac{Z_{s}(t)}{\tau_{o s}} d t \\
& d Z_{0}(t)=\frac{Z_{m}(t)}{\tau_{o m}} d t+\frac{Z_{s}(t)}{\tau_{o s}} d t
\end{aligned}
$$

where $\tau_{\text {om }}$ and $\tau_{\text {os }}$ are time constants representing the rates of transfer of material from the conventional deep lung compartments to the non-clearing sequestration compartment, and $Z_{0}(t)$ is the normalised lung burden at time $t$ in the latter. It is noted here that, unlike Soderholm (at Raleigh), we have chosen not to draw the distinction between what is sequestrated and what is cleared to the lymph nodes. It is assumed (consistent with our decision experimentally to include the mediastinal and hilar lymph nodes in our samples) that the sequestration compartment includes the lymphatic system together with other locations where material may be located within the tissues.

For an exposure time $T$ followed by postexposure time $\Delta \mathrm{T}$, and assuming for the present that $\tau_{\mathrm{om}}=\tau_{\mathrm{os}}$ $=\tau_{\mathrm{o}}$, then the above equations (8) lead to

$$
\begin{aligned}
& \mathrm{Z}_{\mathrm{m}}(\mathrm{T}, \Delta \mathrm{T})=\alpha_{\mathrm{m}} \tau_{\mathrm{m}}{ }^{*}\left[1-\exp \left(-\mathrm{T} / \tau_{\mathrm{m}}{ }^{*}\right)\right] \\
& \mathrm{Z}_{\mathrm{s}}(\mathrm{T}, \Delta \mathrm{T})=\alpha_{\mathrm{s}} \tau_{\mathrm{s}}{ }^{*}\left[1-\exp \left(-\mathrm{T} / \tau_{\mathrm{s}}{ }^{*}\right)\right] \\
& \mathrm{Z}_{0}(\mathrm{~T}, \Delta \mathrm{T})=\left(\int_{0}^{\mathrm{T}+\Delta \mathrm{T}}\left[\mathrm{\tau}_{\mathrm{m}}{ }^{*}\right)\right. \\
& \exp \left(-\Delta \mathrm{T} / \tau_{\mathrm{s}}{ }^{*}\right)
\end{aligned}
$$

where

$$
\tau_{m}^{*}=\tau_{m} \tau_{o} /\left(\tau_{m}+\tau_{o}\right)
$$

and

$$
\tau_{s}^{*}=\tau_{s} \tau_{o} /\left(\tau_{s}+\tau_{o}\right)
$$

from which the overall normalised lung burden for the deep lung may be calculated. Now there is no dependence of the build up of normalised lung burden on the dust level during exposure or on the lung burden which has already accumulated. The results, choosing $\tau_{o}=80$ days empirically and using the same $\alpha$ and $\tau$-values already referred to, are plotted in fig 7 . When they are compared with the experimental data, agreement is seen to be good.

The particular model described is one of several mathematical constructions appropriate to the sequestration concept. As another alternative, for example, Chan et al obtained good agreement with their experimental results for diesel fume by assuming that a fraction of inhaled particles is deposited directly into a compartment from which particles are cleared only slowly. ${ }^{12}$ Our model embodies the important feature that the longer a particle remains in the conventional compartments of the deep lung without being cleared the greater its chance of being sequestrated (and therefore still less the chance of it being cleared). This is believed to be a plausible biophysical hypothesis. New experiments, however, will be required to test its validity, and these are at present being considered.

\section{Discussion}

Interpretation of experiments with living systems in terms of kinetic models (as described in this paper) must be tempered by the inherent limitations imposed by biological variability. This is particularly the case for prolonged chronic exposure studies where the advancing age of the subjects and, possibly, the onset of disease could bring about changes in response. Notwithstanding such reservations, it is clear that the simple kinetic model (based on the Task Group on

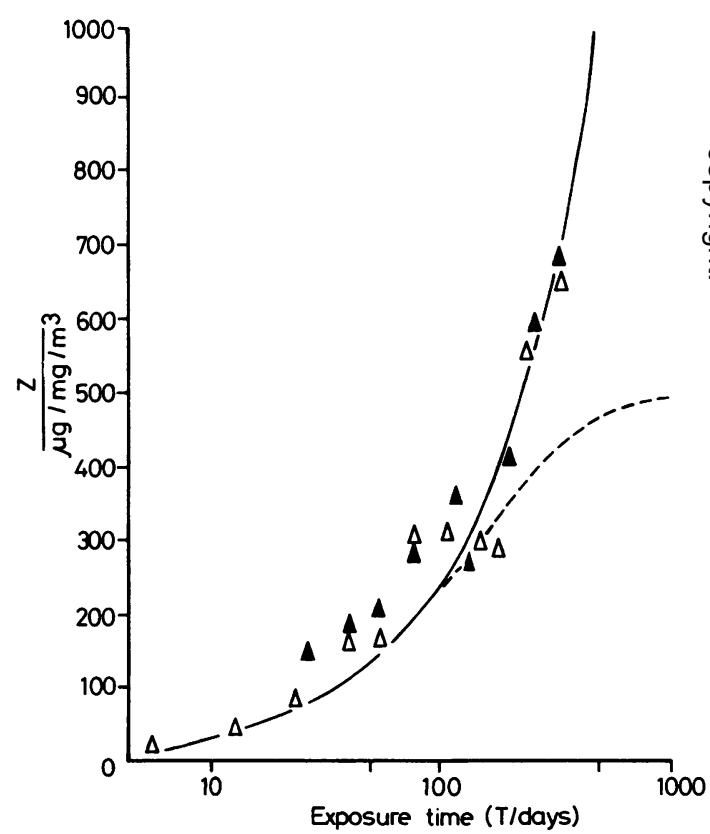

Fig 7 Results of predictions of lung burden during chronic exposure using "sequestration" kinetic model with parameters derived from short term studies with rats inhaling UICC amosite ${ }^{235}$ and $\tau_{0}=80$ days chosen empirically (solid line). Calculations are compared with experimental data for exposure concentrations of 1 and 10 $\mathrm{mg} / \mathrm{m}^{3}$ respectively, and for postexposure time $(\Delta T) 10$ days. Predictions based on simple model are shown, for comparison purposes, in form of dashed line. 
Lung Dynamics ${ }^{1}$ ) for the deposition and clearance of inhaled particulate material fails to account for the steady accumulation of dust in the lung observed during prolonged chronic exposure, not only to UICC amosite as in our experiments but also to other materials as reported elsewhere.

The concept of an overload of clearance (and the implication of impairment of clearance associated with the amount of particulate material accumulated in the deep lung) has been referred to by several workers, based on studies of clearance after short term inhalation exposures. This is reasonable since, for any dust, there must be a lung burden beyond which the lung's defence mechanisms become overwhelmed. Nevertheless, such an overload hypothesis is not consistent with the main trends in lung burden observed during prolonged chronic exposure. The general similarity between our results for asbestos (in Bolton et $^{\mathrm{al}^{5}}$ and the present paper) and those of the General Motors group for diesel fume (in Vostal et $\mathbf{a l}^{\mathbf{1 0}}$ and Chan et $\mathbf{l}^{12}$ ), comparing results for clearance after short term inhalation with those for cumulative lung burden during prolonged chronic exposure, is striking in bringing out this apparent anomaly. (Indeed, it shows up-on closer inspection-even within the data of the short term clearance experiments reported. ${ }^{512}$ ) The overall comparison of our respective experiences for aerosols differing so widely in physical and chemical characteristics is remarkable, and suggests that the observed trends are general in nature and may therefore have even wider relevance.

Both bodies of work indicate that different kinetic models are required to explain the consequences of short term and of chronic exposure respectively. Some further evidence in support of such an idea is available from elsewhere. For example, Klosterkotter and Bunemann noted that clearance in rats of inhaled quartz, where exposure had been interrupted for an extended period and then renewed, was markedly more rapid than for corresponding animals permanently removed from exposure. 9 They postulated that the renewed stimulus of dust challenge serves to further activate clearance mechanisms and to mobilise older dust deposits. Einbrodt too showed that interruption of exposure of rats to quartz had an unfavourable effect on clearance. ${ }^{19}$ Furthermore, he reported some results of readings of $x$-ray films of the lungs of miners suggesting that progression of silicosis was more rapid for those whose occupations had been interrupted for an extended period because of military service, for example, than for corresponding men who had worked continuously. Le Bouffant et al showed that continuing exposure to dust was associated with some inhibition of pneumoconiotic changes in experimental animals. ${ }^{20}$

In relation to the apparent anomaly we have identified between the short term and chronic exposure results, it is therefore one interpretation that overload of clearance associated with high lung burden, which would be apparent after exposure ceases, is - in effect-cancelled out by the sustained stimulus to the lung's defence mechanisms associated with chronic exposure. If such ideas can be shown to be biologically valid it is believed that they will be important in epidemiology relating to various profiles of occupational dust exposure (such as peak versus continuous). They therefore deserve closer inspection.

Of the alternative kinetic models relating to prolonged chronic exposure, the sequestration model provides perhaps the most convincing picture. Indeed, the linearity with time of the rise in cumulative lung burden evident in our own results for amosite and in those of Vostal et al for diesel fume, ${ }^{10}$ as well as those reported for coal quartz dusts by Weller ${ }^{21}$ and recently by Robertson et $a^{22}$ at this institute, may only be explained in this way. For asbestos fibres, it is possible to construct a mechanistic argument for sequestration along the lines that such particles, by virtue of their extreme shape, may be more readily entrapped than compact particles in the fixed tissue of the lung. Morgan et al have given this as one of the reasons that longer fibres appeared to be more difficult to clear than shorter ones in their rat inhalation studies with anthophyllite. ${ }^{23}$ Nevertheless, the more general applicability of the sequestration hypothesis is supported by the other work we have cited, as well as by the results just emerging from our own new inhalation studies with titanium dioxide (to be published in due course). The actual process of sequestration probably involves (a) focal aggregation (in macrophages) within alveolar spaces, particularly in the peribronchiolar region; (b) location within epithelial cells and in the alveolar interstitium (as recently shown by Brody $e$ al $^{2425}$; (c) entrapment in areas of pathological fibrotic change; and (d) collection within the pulmonary lymphatic system. ${ }^{16}$ As described previously, ${ }^{35}$ histological examination of lung tissue taken from animals in experiments with UICC amosite showed no fibrotic change, suggesting that sequestration occurs in the absence of disease. Our results-including the observation of the lack of dose effect on cumulative lung burden-lead us to the conclusion that the mathematical concept of a sequestration compartment reflects the "normal" response of the lung to challenge by airborne dust, and is not therefore primarily the consequence of impairment of the lung's defence mechanisms by that challenge. While the rate of sequestration, as governed in our model by the single parameter $\tau_{\mathrm{o}}$, may be strongly influenced by particle size and shape as suggested, it may also be influenced by the toxicity of the inhaled particulate (since it is well known that transport to the 
lymph nodes is enhanced for more toxic material ${ }^{2627}$ ).

It is important here, while discussing sequestration, to note that clearance processes (including dissolution in body fluids) that have time constants long compared with the relatively short life spans of rats may not be so in relation to human subjects. Caution must therefore be exercised in assuming from our results that lung burdens in people under chronic exposure will simply go on increasing linearly (as our animal results seem to indicate). What might appear as permanent sequestration in rats might, in people, turn out to take the form of a slow clearing compartment. The consequence of this would be that the lung burden in people would eventually level off. On the other hand, however, such a tendency may be offset to some extent by the fact the pulmonary clearance in people is much slower than in rats. ${ }^{16}$

The decision to include the mediastinal and hilar lymph nodes in the biological tissue which was analysed for dust burden was based partly on the fact that it is impossible in practice to separate all lymphatic tissue from the lung and partly on the prior observation that lymph node burdens of UICC amosite (from analysis of tissues taken from previous inhalation studies) were small in relation to overall pulmonary burdens. It therefore follows that the sequestration compartment which we have defined includes the lymphatic system of the lung. It is believed, however, that lymph node accumulation alone is not enough to account for all the sequestrated material but that there must be substantial contributions from other sources (as already suggested).

The main objective of this work has been to investigate the mathematical behaviour of the rat lung system in response to chronic exposure to airborne dust and to look for consistent trends that could lead to a more general kinetic picture. None of the work which has been described sets out to answer directly the many questions which are raised about the underlying biology (such as the mechanisms of clearance overload and sequestration or macrophage processes-recruitment, motility, and phagocytosisin response to the dust challenge or the effects of inflammation and disease). Experimental studies aimed at investigating the detailed cellular response to inhaled mineral dusts, retention in relation to lung morphometry, and pathological response are in progress at this institute and will be reported separately.

Finally, it is appropriate to discuss how what is being learnt about the kinetics of deposition and clearance of inhaled dust may be applied in epidemiology. Perhaps, eventually, this will be one of the most important areas of application of such work. Recently, Smith has described a generalised compartmental model, based on earlier such models but modified to include penetration of dust particles through the alveolar membrane into the interstitium, sequestration, and toxicity effects leading to fibrosis. ${ }^{28} \mathrm{He}$ used it with encouraging success to estimate interstitial dust and fibrosis in silicon carbide workers, taking into account variations in the time profile of worker exposure to quartz. This example serves to show the potential for applying such a kinetic model as an epidemiological tool for relating exposure to dose. The animal studies described in the present paper provide an important basis for the construction of representative models for such applications.

\section{Conclusions}

New inhalation experiments have been carried out to investigate the accumulation of UICC amosite asbestos in the lungs of rats during prolonged chronic exposure. The results are qualitatively consistent with those obtained by workers elsewhere for diesel fume. Several mathematical models for describing the kinetics of deposition and clearance of inhaled relatively non-toxic, insoluble material have been examined. From the evidence which has built up during this and earlier studies, the following main conclusions may be drawn:

(1) During chronic exposure over a wide range of dust concentrations, lung burden scales directly if proportion to the respirable dust exposure leveto Accumulation of lung burden is substantially moret rapid than predicted by the simple kinetic model; indeed, it continues to increase linearly. It is recognised, from the evidence of clearance after short term exposure (as we reported in our earlier paper ${ }^{5}$ ), that the lung's defence mechanisms may be subsequently impaired by the amount of dust accumulated. Such an overload, however, does not account for the observed results for prolonged chronic exposure where, it is speculated, clearance may be sustained by the continuing dust challenge. It seems therefore that, especially for high lung burden, different kinetic models are required for prolonged continuous exposure than for clearance after exposure has ceased. It is suggested that the observed trend represents "normal" (as opposed to impaired) lung response.

(2) The most plausible alternative kinetic model to explain these results is the one which invokes a sequestration compartment in the deep lung from which material is either cleared extremely slowly or, possibly, not at all. No distinction is drawn at this stage between what is sequestrated by encapsulation in the fixed tissue of the lung and that which is transported to the lymph nodes. The rate at which material is transferred to the sequestration compartment from the other compartments of the deep lung is believed to 
be influenced by the size and shape of the inhaled particles, by their toxicity, and by the presence or otherwise of fibrosis or lipidosis. The particular sequestration model favoured is one that embodies the important feature that, the longer a particle remains in the lung without being cleared, the more likely it will be sequestrated (and therefore less likely to be cleared).

(3) Although all of our own experiments reported so far have been carried out with rats using UICC amosite, evidence from elsewhere suggests that broadly similar behaviour is to be expected for other inhaled particulate material. Therefore it is believed that many of the trends which have been reported are general in nature and, as such, deserve closer inspection.

(4) Workers elsewhere have indicated the potential for the practical application of ideas such as those contained in this paper to epidemiology and toxicology. Before such applications become reality, however, further studies of inter-species and interdust effects are required. Until these are carried out and a broader range of experimental data is available, any general conclusions arising out of such work should remain tentative.

We thank the Asbestosis Research Council (UK) for their financial support of this work; also Christine McMillan, Carol McIntosh, George Borzucki, Michael Whittington, and Graeme Hughson for their invaluable technical contributions.

\section{References}

${ }^{1}$ Task Group on Lung Dynamics. Deposition and retention models for internal dosimetry of the human respiratory tract. Health Physics 1966;12:173-207.

${ }^{2}$ Morgan A. Evans JC, Holmes A. Deposition and clearance of inhaled fibrous minerals in the rat. Studies using radioactive tracer techniques. In: Walton WH, ed. Inhaled particles IV. Oxford: Pergamon Press, 1977:259-72.

${ }^{3}$ Middleton AP, Beckett ST, Davis JMG. A study of the short term retention and clearance of inhaled asbestos by rats, using UICC reference samples. In: Walton WH, ed. Inhaled particles IV. Oxford: Pergamon Press, 1977:247-57.

${ }^{4}$ Middleton AP, Beckett ST, Davis JMG. Further observations on the short term retention and clearance of asbestos by rats using UICC reference samples. Ann Occup Hyg 1979;22:141-52.

${ }^{5}$ Bolton RE, Vincent JH, Jones AD, Addison J, Beckett ST. An overload hypothesis for pulmonary clearance of UICC amosite fibres inhaled by rats. Br J Ind Med 1983;40:264-72.

${ }^{6}$ Wagner JC, Berry G, Skidmore JW, Timbrell V. The effects of the inhalation of asbestos in rats. Br J Cancer 1974;29:252-69.

${ }^{7}$ Davis JMG, Beckett ST, Bolton RE, Collings P, Middleton AP. Mass and number of fibres in the pathogenesis of asbestosrelated disease in rats. Br $J$ Cancer 1978;37:673-88.

${ }^{8}$ Ferin J. Observations concerning alveolar dust clearance. NY Acad Sci 1972;200:66-72.

${ }^{9}$ Klosterkotter W. Bunemann G. Animal experiments on the elimination of inhaled dust. In: Davies CN. ed. Inhaled particles and vapours. Oxford: Pergamon Press, 1961:327-37.

${ }^{10}$ Vostal JJ, Schreck RM. Lee PS. Chan TL. Soderholm SC. Deposition and clearance of diesel particles from the lung. In: Lewtas S. ed. Toxicological effects of emissions from diesel engines. New York: Elsevier Science Publishing Company. 1982:143-59.

${ }^{11}$ Beckett ST. The generation and evaluation of UICC asbestos in animal exposure chambers. Ann Occup Hyg 1975:18:187-98.

${ }^{12}$ Chan TL. Lee PS. Hering WE. Pulmonary retention of inhaled diesel particles after prolonged exposures to diesel exhaust. Fundamental and Applied Toxicology 1984;4:624-31.

${ }^{13}$ Lauweryns JM. Baert JH. Alveolar clearance and the role of the pulmonary lymphatics. Am Rev Respir Dis 1977;115:625-83.

${ }^{14}$ Brain JD. Pulmonary clearance of inhaled particles: introductory statement. In: Brain JD. Proctor DF. Reid LM, eds. Respiratory defense mechanisms. New York: Dekker, 1977. (Preface to vol 2.)

${ }^{15}$ Morgan A. Holmes A. Davison W. Clearance of sized glass fibres from the rat lung and their solubility in vivo. Ann Occup $H y g$ 1982:25:317-31.

${ }^{16}$ Snipes MB, Boecker BB, McClellan RO. Retention of monodisperse aluminosilicate particles inhaled by dogs, rats and mice. Toxicol Appl Pharmacol 1983;69:345-62.

${ }^{17}$ Bailey MR, Hodgson A, Smith H. Respiratory tract retention of relatively insoluble particles in rodents. Journal of Aerosol Science (in press).

${ }^{18}$ Bailey MR. Fry FA, James AC. Long-term retention of particles in the human respiratory tract. Journal of Aerosol Science (in press).

${ }^{19}$ Einbrodt HJ. The influence of dust elimination and the effects on the development of pneumoconiosis. In: Shapiro HA, ed. Pneumoconiosis: proceedings of the international conference (Johannesburg). Cape Town: Oxford University Press, 1970:299-305.

${ }^{20}$ Le Bouffant L, Daniel H. Martin JC. Quartz as a causative factor in pneumoconiotic lesions in coal miners. Luxembourg: Commission of the European Communities, 1977. (Industrial health and medicine series No 19.)

${ }^{21}$ Weller $\mathrm{W}$. The relationship between duration of dust inhalation of a coal-quartz mixture and dust retention, lung function and pathology on rats. In: Walton WH, ed. Inhaled particles III. Oxford: Pergamon Press, 1971:337-45.

${ }^{22}$ Robertson A, Bolton RE, Chapman JS, et al. Animal inhalation experiments to investigate the significance of high and low percentage concentrations of quartz in coalmine dusts in relation to epidemiology and other biological tests. Edinburgh: Institute of Occupational Medicine, 1984. (No TM/84/5.)

${ }^{23}$ Morgan A, Talbot RJ, Holmes A. Significance of fibre length in the clearance of asbestos fibres from the lung. $\mathrm{Br} J$ Ind $\mathrm{Med}$ 1978;35:146-53.

${ }^{24}$ Brody AR, Hill LH, Adkins B, O’Connor RW. Chrysotile asbestos inhalation in rats: deposition pattern and reaction of alveolar epithelium and pulmonary macrophages. Am Rev Respir Dis 1981;123:670-9.

${ }^{25}$ Brody AR, Roe MW, Evans JE, Davis GS. Deposition and translocation of inhaled silica in rats. Quantification of particle distribution, macrophage participation and function. Lab Invest 1982:47:533-42.

${ }^{26}$ Einbrodt HJ, Kinny H, Kortemme H. Quantitative Untersuchungen uber den lymphtransport von Blei aus der menschlichen Lunge. Arch Hyg 1969:153:105-8.

${ }^{27}$ Bruch J, Hilscher W, Kramer U. Pathogenicity to animals of fine dusts from Ruhr mines. In: Walton WH, ed. Inhaled particles IV. Oxford: Pergamon Press, 1977:373-8.

${ }^{28}$ Smith TJ. Development and application of a model for estimating alveolar and interstitial dust levels. Ann Occup Hyg (in press). 